\section{A subscription service that pays back}

Ordo isn't your average oral health company: just launched as part of the Henry Schein Dental Business Solutions portfolio, Ordo offers a subscription service with a difference. Not only does the dentist profit from patient purchases of Ordo Sonic+ toothbrushes, but they also receive an additional $10 \%$ commission on all refills when the patient subscribes to Ordo.

Ordo is on a mission: to revolutionise the oral care industry and transform the way that patients look after their teeth in between visits to the practice. Ordo believes that oral hygiene is about using the correct products at the right time - and replacing them when needed is essential to maintaining a healthy smile.

How does it work? Ordo provides an affordable subscription service for the patient's complete oral health needs. This includes a FREE starter kit containing toothpaste, 18 interdental brushes, mint floss, mouthwash concentrate, reuseable mouthwash bottle and a USB adapter (just cover shipping of $£ 3.95$ ). Then refills are delivered straight to the door as often as the patient wishes, all for just $£ 15$ for a $2-3$ month supply. The subscription can be customised or cancelled at any time.

Practices sell the brush for a profit upfront and then receive recurring revenue for their patients' oral care refills with a $10 \%$ commission for every refill sold - the system couldn't be simpler.

Join the Oral Care Revolution today by visiting https://www.hsbusinesssolutions. co.uk/ordo or emailing info@henryschein. co.uk.

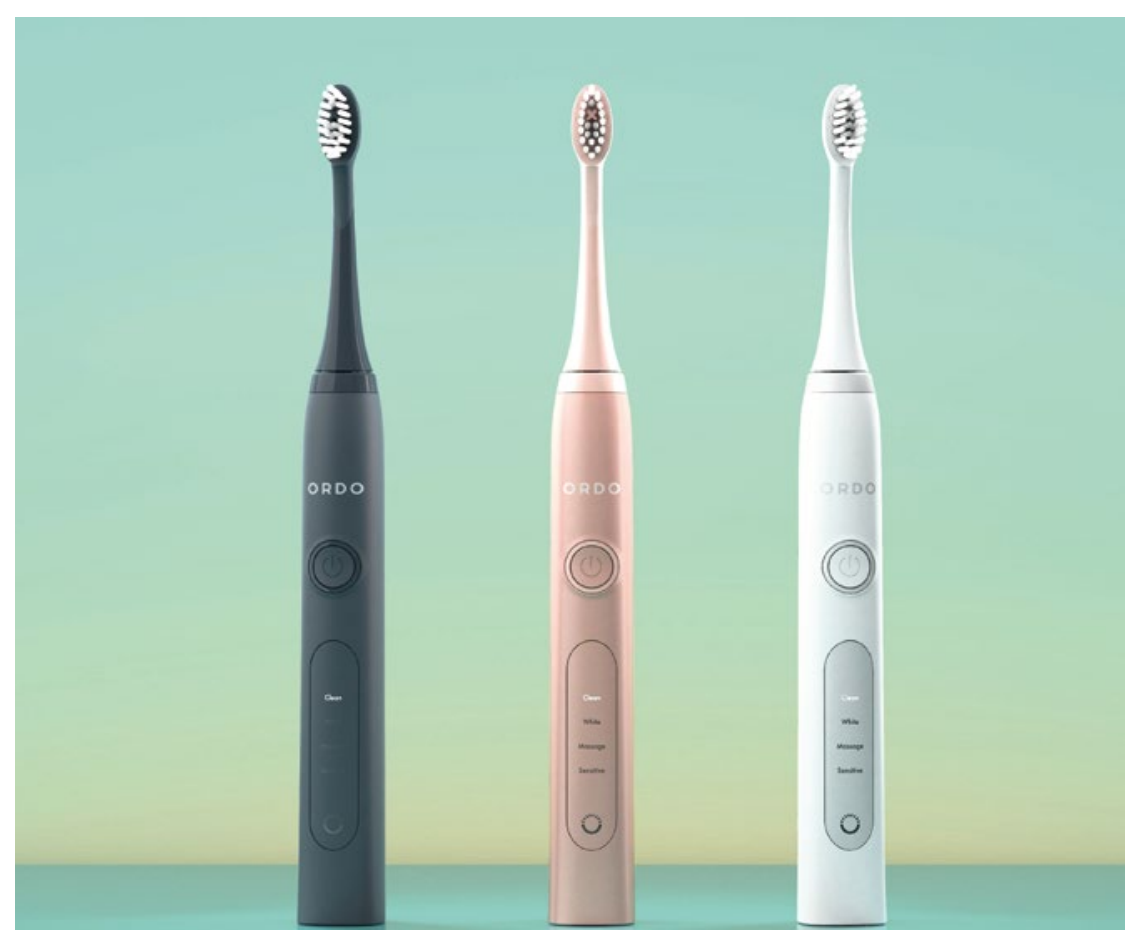

\title{
Fresh solutions for specialists and general dentists
}

Specialists and general dentists will find out how to upgrade their dentistry when they visit the COLTENE stand at the BDIA Dental Showcase. High-quality dentistry requires high-quality tools and materials. COLTENE has products for everything from endodontics to restorative treatment and delegates will see the full range at Showcase, including new additions.

The HyFlex EDM file system, for efficient root canal therapy, was expanded this year with the Glidepath file15/.03, for curved and narrow canals. Also new, COLTENE's BRILLIANT COMPONEER for chairside restorations joins favourites like BRILLIANT EverGlow. Be sure to stop by stand D40 at the NEC in October!

To find out more visit www.coltene. com, email info.uk@coltene.com or call 01444235486 .

\section{Free CPD portal focused on}

\section{enamel erosion}

Regenerate has launched a new learning portal focussed on enamel erosion which provides free CPD for dental professionals. The Learning Resources portal is accessed via its website www.regeneratenr5.co.uk/ pages/learning-resources.

A core feature of the portal is five one hour CPD modules, which cover different aspects of erosive enamel wear:

- New Perspectives on Enamel Erosion: From Diagnosis to Prevention

- Enamel Erosion: Tooth Wear

- Enamel Erosion

- Enamel Erosion: Diagnosis

- Enamel Erosion: Unilever Lunch and Learn.

Each of the five free e-learning modules confers an hour of verifiable CPD, and is accredited by Healthcare Learning. A downloadable certificate is provided on completion of each of the modules.

The portal features a number of clinical papers about the technology behind the REGENERATE Enamel Science range which is the first oral care regime with NR-5 technology able to regenerate enamel mineral. Eighty-two percent of enamel mineral is regenerated after three days (acts on early invisible stages of enamel erosion. Helps to regenerate enamel by restoring its mineral content and micro hardness with regular use. Clinically proven). A summary document containing all the studies can also be downloaded.

Videos demonstrating the mode of action of the REGENERATE Enamel Science Advanced Toothpaste and Advanced Enamel Serum can also be viewed on the portal.

In addition, a downloadable guide to diagnosing enamel erosion in clinic as well as a range of patient resources including three patient brochures, clinic posters and leaflets about enamel erosion are available. Dental professionals can also link to the Erosive Tooth Wear Foundation and access a range of videos featuring Professor David Bartlett explaining enamel erosion.

The portal is free for all dental professionals: https://www.regeneratenr5.co.uk/ pages/learning-resources. 